Communications in Physics, Vol. 20, No. 3 (2010), pp. 271-276

\title{
ON THE YANG - MILLS GRAVITY
}

\author{
NGUYEN VIEN THO AND NGUYEN QUOC HOAN \\ Hanoi University of Technology
}

\begin{abstract}
The parallel between Yang-Mills theory and General Relativity is an attractive topic that motivated a lot of interesting researches. Various versions of the gauge theory of gravity were proposed which provided a deep understanding of such basis concepts as the geometry of spacetime, the connection between symmetry and dynamics. In this work we consider the problem of interaction between intrinsic degrees of freedom of particles with the Lorentz gauge field solution that has the same singular feature as for the Schwarzschild solution of General Relativity. The effective potential for a particle in the Schwarzschild-like Lorentz gauge field was investigated in comparison with Einstein and Newtonian theories of gravity. The equations of motion for particles allows planar motions that can be solved numerically. We have shown that there exit the Keplerlike orbits which correspond to certain sets of parameters and initial conditions. The obtained results can be regarded as an experimental evidence for the existence of the "internal Lorentz symmetry" of point particles that is analogue to internal unitary symmetries.
\end{abstract}

\section{INTRODUCTION}

It is well known that the Yang-Mills theory became a general framework to formulate theories of fundamental interactions. The idea of Yang and Mills [1] is to extend the SU(2) internal symmetry to the local $\mathrm{SU}(2)$ one: If we want to make a physical theory invariant under local symmetry transformations, it is necessary to introduce compensating fields. The idea was then applied to other unitary internal symmetries (SU(3), etc...). Utiyama [2] observed that the whole idea of principle of equivalence of General Relativity can be reformulated in accordance with the philosophy of Yang-Mills theory: the gravitational field can be interpreted as compensating field that cancel all the unwanted effects of local space-time transformations. Utiyama made a direct generalization of the Yang-Mills scheme for the $\mathrm{SU}(2)$ group to the Lorentz group. Various versions of gauge theory of gravitation were developed which provided a deep understanding of geometrical structure of space-time (Poincare gauge theory [3-5], Weyl gauge theory [6-8], etc...), and possibility of unifying gravity with other interactions (supergravity, see, for example, [9]).

Drawing on the parallel between General Relativity and Yang-Mills theory, Singleton has introduced an interesting ansatz and discovered an exact Schwarzschild-like solution for Yang-Mills theories [10] that exhibits the property of confinement by an event horizon. In the present paper we show that the pure gauge case of the mentioned Schwarzschildlike solution can be converted into a static gauge field for the Lorentz group, and then we investigate the motion of particles interacting with this gauge configuration. In Sec. II we recall the Singleton's solution and describe how can construct the corresponding gauge field for the Lorentz group. In Sec. III, basing on the system of equations that describes 
the interaction of intrinsic degrees of freedom of particle with Lorentz gauge field [11], the equations of motion for particles are derived and investigated. We show that the system of equations allows planar motions and determine the conditions of them. In Sec. IV planar motions are investigated. We compare the effective potential for particles in our approach with that of the Newtonian gravity and the General Relativity. By trying to solve numerically the equations of motion with chosen parameters, we show that there exists the possibility of Kepler-like orbits. Some discussions on the obtained results are given in Sec. V.

\section{SCHWARZSCHILD-LIKE SOLUTION FOR YANG-MILLS THEORIES}

Exact solutions to nonlinear field theories, and in particular, Yang-Mills theories, are usually found by making some guess as to the form of the solution and insert it into the field equations to see if it solves them. Let us consider the model of an $S U(2)$ gauge field coupled to a massless scalar triplet. The Lagrangian for this system is

$$
L=-\frac{1}{4} F^{\mu \nu a} F_{\mu \nu}^{a}+\frac{1}{2} D^{\mu}\left(\phi^{a}\right) D_{\mu}\left(\phi^{a}\right),
$$

where

$$
F_{\mu \nu}^{a}=\partial_{\mu} A_{\nu}^{a}-\partial_{\nu} A_{\mu}^{a}+g \epsilon^{a b c} A_{\mu}^{b} A_{\nu}^{c},
$$

and

$$
D_{\mu}\left(\phi^{a}\right)=\partial_{\mu} \phi^{a}+g \epsilon^{a b c} A_{\mu}^{b} \phi^{c} .
$$

The Yang-Mills equivalent of the Schwarzschild solution in General Relativity was discovered by Singleton [10], by using the ansatz

$$
A_{i}^{a}=\epsilon^{a i j} \frac{r^{j}}{g r^{2}}[1-K(r)], \quad A_{0}^{a}=\frac{r^{a}}{g r^{2}} J(r), \quad \phi^{a}=\frac{r^{a}}{g r^{2}} H(r),
$$

where

$$
K(r)=\frac{C r}{1-C r}, \quad J(r)=\frac{B}{1-C r}, \quad H(r)=\frac{C}{1-C r},
$$

$A, B, C$ are arbitrary constants. The non-Abelian gauge potentials and the scalar fields of the form (4), (5) solve the Yang-Mills field equations that correspond to the Lagrangian (1) with the only constraint $A^{2}-B^{2}=1$.

We are interested in the pure gauge case, where there is no scalar field, $H(r)=0$, which implies $A=0$. The condition $A^{2}-B^{2}=1$ requires that $B= \pm i$, and the solution becomes

$$
K(r)=\frac{C r}{1-C r}, \quad J(r)=\frac{ \pm i}{1-C r}, \quad H(r)=0 .
$$

Here a remarkable fact is that one has a static complex solution for the $\mathrm{SU}(2)$ Yang-Mills field equations. However it is not strange. Another complex solution for the SU(2) YangMills theory was discovered earlier by Hsu [12], and then was converted into a real gauge field for the Lorentz group by Wu and Yang [13].

Therefore, in accordance with the procedure of Wu and Yang in [13], the gauge field solution, given by Eqs. (4), (6) can be converted into a real gauge for the Lorentz group. Further we can interpret it as a sourceless gravitational field configuration in the YangMills approach of gravity, which is spherically symmetric and caused by a mass located at 
the coordinates origin. It is interesting to investigate how does a test particle move in the Yang-Mills field given by Eqs. (4), (6).

\section{MOTION OF PARTICLES IN SCHWARZSCHILD-LIKE YANG-MILLS FIELD}

It is known that the interaction of the isotopic degrees of freedom of a color particle with the $S U(2)$ gauge field is described by the Wong's equations [14]. The equations of motion for particles in a Lorentz gauge field were derived in the paper [11] which can be considered as a generalization of the Wong's equations. Suppose that that a point-like particle must have the "intrinsic Lorentz symmetry" and therefore carries corresponding intrinsic degrees of freedom that is characterized by the complex isospin vector $I_{a},(a=$ $1,2,3$ ). The interaction of a particle (with the rest mass $m$ ) with the gauge field $A_{\mu a}$ is described by the following system of equations [11]

$$
\begin{cases}m \frac{d}{d \tau}\left(\frac{\dot{x}_{\mu}}{|\dot{x}|}\right)=G F_{\mu \nu a}(x) I_{a} \dot{x}^{\nu}+(c . c .), & (a) \\ \dot{I}_{a}=G \epsilon_{a b c} I_{b} A_{\mu c}(x) \dot{x}^{\mu}, & (b) \\ \dot{I}_{a}^{*}=G \epsilon_{a b c} I_{b}^{*} A_{\mu c}^{*}(x) \dot{x}^{\mu}, & (c)\end{cases}
$$

where $F_{\mu \nu a}(x)$ is the gauge field intensity, on the right-hand side of (6a) we have denoted by (c.c.) the complex conjugate term of the first one, the indexes $a, b, c$ take values $1,2,3$. Remark that although we deal with the complex isospin vector and the complex gauge field, in the equation (6a) the right-hand side is a real quantity. In a given gauge configuration the motion of particles in the outer space is completely determined.

Insert the gauge field of the form (4), (6) into the system (7), after some manipulations we obtain the equation that governs the motion of particle in the outer space

$$
\begin{gathered}
\frac{d}{d t}\left(\frac{m \mathbf{v}}{\sqrt{1-v^{2}}}\right)=\mathbf{F}_{1}+\mathbf{F}_{2}, \\
\mathbf{F}_{1}=2\left(\frac{J-1}{r}\right)^{\prime}(\mathbf{Y} \cdot \mathbf{n}) \mathbf{n}+2 \frac{K(J-1)}{r^{2}}[\mathbf{Y}-(\mathbf{Y} . \mathbf{n}) \mathbf{n}], \\
\mathbf{F}_{2}=-2\left(\frac{K^{2}-1}{r^{2}}\right)(\mathbf{X} . \mathbf{n})(\mathbf{v} \times \mathbf{n})+2\left(\frac{K^{\prime}}{r}\right)[(\mathbf{v} \times \mathbf{X})-(\mathbf{X} . \mathbf{n})(\mathbf{v} \times \mathbf{n})],
\end{gathered}
$$

and the equations for the time evolution of the complex isospin vector

$$
\begin{gathered}
\dot{\mathbf{X}}=-\left(\frac{J-1}{r}\right)(\mathbf{Y} \times \mathbf{n})+\left(\frac{1-K}{r}\right)[(\mathbf{X} . \mathbf{n}) \mathbf{v}-(\mathbf{X . v}) \mathbf{n}], \\
\dot{\mathbf{Y}}=\left(\frac{J-1}{r}\right)(\mathbf{X} \times \mathbf{n})+\left(\frac{1-K}{r}\right)[(\mathbf{Y} . \mathbf{n}) \mathbf{v}-(\mathbf{Y} . \mathbf{v}) \mathbf{n}] .
\end{gathered}
$$

In Eqs. (8), (9) by $\mathbf{X}$ and $\mathbf{Y}$ we have denoted the real and the imaginary parts of $\mathbf{I}$, $\mathbf{I}=\mathbf{X}+i \mathbf{Y}$. From Eq. (6) it is not difficult to show that $\mathbf{X}$ and $\mathbf{Y}$ depend on time, but $|\mathbf{X}|,|\mathbf{Y}|$ and $(\mathbf{X} . \mathbf{Y})$ are constants of motion.

The system of Eqs. (8), (9) allows planar motions that take place under the following conditions: at the initial moment the vector $\mathbf{X}$ is normal to the plane spanned by the vectors $\mathbf{r}_{0}$ and $\mathbf{v}_{0}$, while the vector $\mathbf{Y}$ is in this plane. So, we have initially $(\mathbf{X} . \mathbf{n})=$ 
$(\mathbf{X} . \mathbf{v})=0,(\mathbf{X} . \mathbf{Y}))=0$, and the equation (9) becomes $\dot{\mathbf{X}}=-[(J-1) / r](\mathbf{Y} \times \mathbf{n})$, which means $\dot{\mathbf{X}} \| \mathbf{X}$, the direction of the vector $\mathbf{X}$ does not change. Since $|\mathbf{X}|$ is constant of motion, we have further $\mathbf{X}$ being a conserved vector normal to the plane of motion. Denoting by $\mathbf{M}$ the total momentum vector, we have $(\mathbf{M} . \mathbf{n})=-2(\mathbf{X} . \mathbf{n})$. Therefore we can use the system of coordinates in which the total momentum vector $\mathbf{M}$ (and also $\mathbf{X}$ ) directs along the $z$-axis, so the motion is in the plane $(x, y)$. For the second isospin vector $\mathbf{Y}$, from the equation (9) we see that the conservation of the isospin vector $\mathbf{X}$ leads to the following consequence: $(\mathbf{Y} \times \mathbf{n})=0$, that is $\mathbf{Y} \| \mathbf{n}$, or $\mathbf{Y}=Y \mathbf{n}(Y \equiv|\mathbf{Y}|)$.

With such two isospin vectors we see that the force vector (the right-hand side of the equation (8)) has no component in the $z$-axis normal to the plane of motion $(x, y)$, that is, the motion maintains always in this plane. For simplicity, we will describe planar orbits in the non-relativistic limit in which the equation (8) has the following form:

$$
m \dot{\mathbf{v}}=\chi_{2}\left[\frac{J^{\prime}}{r}-\frac{J-1}{r^{2}}\right] \mathbf{n}-\chi_{1}\left(\frac{K^{\prime}}{r}\right) \mathbf{u},
$$

where we have introduced the constants $\chi_{1} \equiv 2 X, \chi_{2} \equiv 2 Y$, the vector $\mathbf{u}$ is defined by the formula $X \mathbf{u}=(\mathbf{v} \times \mathbf{X}), \mathbf{u}$ is in the plane $(x, y)$ and normal to $\mathbf{v}$.

\section{CONSIDERATION OF PLANAR MOTIONS}

The two-dimensional motion described by Eq. (10) corresponds to the Lagrangian that is written in polar coordinates as follows

$$
L=T-U=\frac{m}{2}\left(\dot{r}^{2}+r^{2} \dot{\theta}^{2}\right)+\chi_{1} K(r) \dot{\theta}+\chi_{2} \frac{J(r)-1}{r} .
$$

The effective potential of the equivalent one-dimensional problem is defined by

$$
V_{e f f}(r)=\frac{m}{2}\left(\frac{j-\chi_{1} K}{m r}\right)^{2}-\chi_{2}\left(\frac{J-1}{r}\right) .
$$

Investigating $V_{\text {eff }}(r)$ in Eq. (12) with the functions $J(r)$ and $K(r)$ defined by Eq. (6), we see that the case of positive sign in the function $J(r)$ must be excluded to have an acceptable potential. The potential depends on the parameters $C, \chi_{1}, \chi_{2}$ and has the form

$$
V_{e f f}(r)=\frac{\left(\chi_{2}-j \chi_{1} C\right)}{r(1-C r)}+\frac{j^{2}}{2 r^{2}}+\frac{1}{2} \cdot \frac{\chi_{1}^{2} C^{2}}{(1-C r)^{2}},
$$

where $j$ is the magnitude of the conserved total angular moment of particle. It is well known that the effective potential gives qualitative characteristics of motion for a test particle in a spherically symmetrical gravitational center. The effective potential and the characteristics of motion for a particle in the Schwarzschild space-time in the Einstein's theory of gravity, in comparison with Newtonian gravity, were presented in many places, see, for example [15]. Using the same symbols and units, as in [15], for relative quantities, we plot the potential (13) together with that of the Newtonian theory

$$
V_{e f f}^{(N)}=-\frac{M}{r}+\frac{L^{2}}{2 r^{2}}
$$


and General Relativity

$$
V_{e f f}^{(G R)}=\left[\left(1-\frac{2 M}{r}\right)\left(1+\frac{L^{2}}{r^{2}}\right)\right]^{1 / 2}
$$

The graphs are given in Fig. 1, where for the potentials we take the values of the parameters as $C=1 / 2 M ; L=4.33 ; j=4.53 ; \chi_{1}=4 . ; \chi_{2}=18.5$. It is seen from the graphs that at radii that is larger than $10 M$ test particles behave in the same manner. Therefore for the potential in our approach there exists the Kepler-like orbits. An example of orbits for a test particle in the Schwarzschild-like Yang-Mills field is shown in Fig. 2, which is obtained by solving numerically the equations of motion for particle. We have used the "MATHEMATICA" programming package for the Runge-Kutta method, the initial conditions for particle's motion are $r_{0}=2.0, v_{0}=0.2 ; \theta_{0}=0 ; \dot{\theta}_{0}=\pi / 4$. The orbit in the presented figure is plotted with the interval of evolution parameter $t=25$.

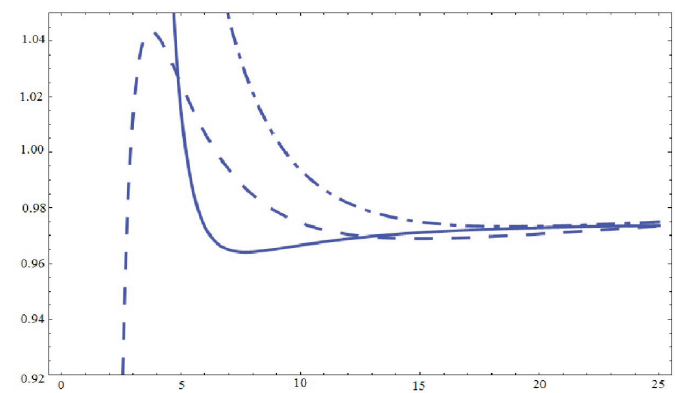

Fig. 1. The effective potential in the Schwarzschild space-time in the Einstein's theory of gravity (dot-dashed curve), Newtonian gravity (dashed curve) and Schwarzschild-like Yang-Mills (solid curve)

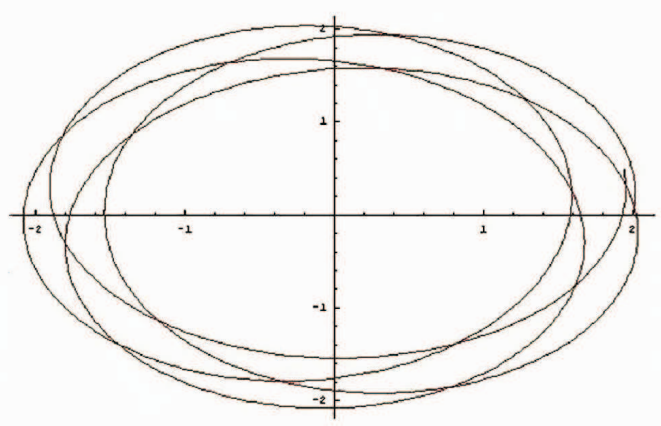

Fig. 2. The orbits for a test particle in the Schwarzschild-like Yang-Mills field 


\section{DISCUSSION}

The main purpose of this work is to find implications of such fundamental concepts as local symmetry principle, its extension from unitary to space-time symmetries, to compare effects from the Yang-Mills approach and the geodesic motion in curve space-time. Here the problem of motion for a test particle in the Schwarzschild-like gravitational field is examined with the Yang-Mills approach: the gravitational configuration is a Lorentz gauge field, and particles interact with the gauge field in the same manner as the color charges do with the $\mathrm{SU}(2)$ gauge field. The obtained results show that our approach is equivalent to Newtonian theory and General Relativity at radii that is larger than 10M.

The differences between the approaches (Newtonian, Einstein and Yang-Mills) are

at small radii. We recall that in our approach, like for Wong's equations, the equations of motion is a classical limit of the quantum equations. Therefore, such effects, as tunnelling across potential barrier at the Schwarzschild radius, can not described by our approach. This kind of effects that concerns to the black hole concept can be described only by Einstein's General Relativity.

This work was supported by the National Foundation for Science and Technology Development, the Grant $N^{\circ} 103.03 .106 .09$.

\section{REFERENCES}

[1] C. N. Yang, R. Mills, Phys. Rev. 96 (1954) 191.

[2] Utiyama P., Phys. Rev. 101 (1956) 1597.

[3] T. W. B. Kibble, J. Math. Phys. 2 (1961) 212.

[4] P. Held Von der Heyde, D. Kerlick, J. Nester, J. Rev. Mod. Phys. 48 (1976), 393.

[5] K. Hayashi, T. Shirafuji, Phys. Rev. D19 (1979) 3524.

[6] A. Bragman, Prog. Theor. Phys. 49 (1973) 667.

[7] J. Charap, W. Tait, Proc. Roy. Soc. A340 (1974) 249.

[8] M. Kasua, Nuovo. Cim. B28 (1975) 127.

[9] J. Wess, J. Bagger, Supersymmetry and Supergravity, Princeton University Press, New Jersey, 1982.

[10] D. Singleton, Phys. Rev. D51 (1995) 5911.

[11] Nguyen Vien Tho, J. Math. Phys. 49 (2008) 062301.

[12] J. P. Hsu, Phys. Rev. Lett. 36 (1976) 646.

[13] T. T. Wu, C. N. Yang, Phys. Rev. D13 (1976) 3233.

[14] S. K. Wong, Nouvo Cimento A65 (1970) 689.

[15] C. W. Misner, K. S. Thorn, and J. A. Wheeler, Gravitation, W. H. Freedman and Company, San Francisco, 1973. 